Historic, Archive Document

Do not assume content reflects current scientific knowledge, policies, or practices. 




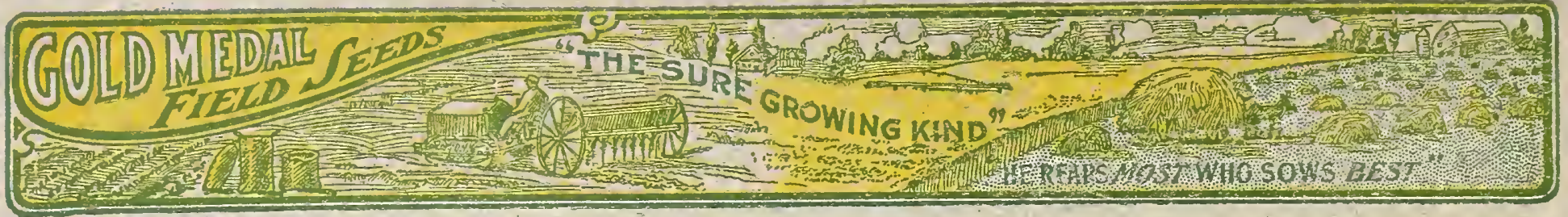

Subject to immediate accoptance and market changes we Guote f. o. b. Louisville

FEB. 25,1927

\section{SPECIAL LOTS}

\begin{tabular}{|c|c|c|c|c|}
\hline & WHITE BLOSSOM & Cwt. & TIMOTHY & Cort. \\
\hline 97\% Pure & SWEET CLOVER & $\$ 10.00$ & Small Grain, Bright Color & $\$ 4.50$ \\
\hline ure & $\begin{array}{l}\text { YELLOW BLOSSOM } \\
\text { SWEET CLOVER } \\
\ldots \ldots\end{array}$ & $\$ 11.00$ & $\begin{array}{c}\text { PASTURE MIXTURE } \\
\text { Contains Timothy, Redtop, Alfalfa, Alsike, etc. }\end{array}$ & $\$ 5.00$ \\
\hline
\end{tabular}

SAMPLES FURNISHED ON APPLICATION

\section{RED CLOVER Girt.}

Gold Medal, Domestic . . \$42 00

Gold Medal, French . . 3200

Choice ....... 3100

Royal $: \ldots 2850$

Special small grain ... 2500

MAMMOTH or SAPLIN CLOVER

Gold Medal . . . . . 4450

Choice ... . 4200

Special small grain ... . 3000

\section{ALSIKE}

Gold Medal . . . . . 3750

Choice . . . 3400

Special contains spininkling red clover . 2500 ALFALFA

Gold Medal : . . . . 2000

Choice ... . . 1850

GRIMMS ALFALFA

Biackfoot, in 30 and $60 \mathrm{lb}$. bags, sealed and certified by Seed

Commissioner Idaho . . 4000 SWEET CLOVER

Grundy Co. W. B. Biennial . 1400 Gold Medal W. B. Biennial . 1350 Choice W. B. Biennial . . 1250 White Biennial, unhulled . 800 Gold Medal Yellow Biennial 1400

Choice Yellow Biennial . 1300 CRIMSON CLOVER

Gold Medal . . . . . . 1500 JAPAN CLOVER

Choice Recleaned . . . . 1000 WHITE CLOVER

Gold Medal . . . . . . 3500 Special ...... 2000
ALSIKE \& TIMOTHY Cwt.

Choice ........ $\$ 900$

Special . . . . 800

TIMOTHY

Gold Medal ..... 655

Abe ....... 625

Choice . . . . 600

Royal . $\ldots 550$

RED TOP

Gold Medal . . . . . 2700

Abe ....... 2600

Choice ...... 2450

Royal ........ 2300

ORCHARD GRASS

Gold Medal ...... 1500

Choice ....... 1400

Royal . . . . 1300

KY. BLUE GRASS

Gold Medal ...... 2200

Choice...... 2050

Royal ....... 1900

\section{RAPE SEED}

Dwarf Essex ... . 700

WINTER OR HAIRY VETCH

$99 \%$ Pure ..... 1650 GERMAN MILLET

Cult Tenn. ..... 370

(On account of the low price of Tennessee

Millet we are not handling any other grade) CANE SEED

Amber ....... 350

Orange..... 325

Redtop . $\ldots 300$

SEED SOWERS

Cyclone each \$1 75 Doz. \$18 00

Horn each 75 Doz. 750
LAWN GRASS Cwt

Gold Medal ..... . $\$ 3000$

Cherokee . . . . 2500

"Shady Place" ..... 4000

Put up in muslin bags printed in two colors, $1 \mathrm{lb}$. 3 lb. 5 lb. net.

Above grades in bulk $\$ 3.00 \mathrm{cwt}$. less

\section{SOY BEANS Bu.}

Early Yellow, Manchu or Midwest . . 225

Mammoth Yellow .... 200

Black Ebony ...... 265

Black Wilson, Laredo or Virginia . . . 285

\section{COW PEAS}

Whipporwill .... Ask

New Era . . . . .

Mixed ..... SPrices

\section{MISCELLANEOUS Cwt.}

Bermuda Grass . . . . 3500

Buckwheat .... 375

Sudan Grass . . . . . 700

Tall Meadow Oat Grass . . 1250

BARLEY

Bu.

Bearded Winter.... 100

\section{OATS}

Winter Turf ... . . 85

Burt ....... 80

White ....... 75

Feed Oats . . . . . . 68

\section{SEED CORN}

Reid's Yellow Dent . . . 225

Boone Co. White . . . 225

Johnson Co. White : . 225

Hickory King (White) ․ 225

Shipment prompt or April 1st to 15th

ORDERS FOR LESS THAN BAG LOTS WILL BE CHARGED FOR AT 500 PER 100 Lbs. BIGHER TEAN LIST PRICES.

DISCLAIMER-The Louisville Seed Company gives no warranty ezpress or implied, as to degcription, quality or any other matter of any seeds it sells and will not be responsible for the orop.

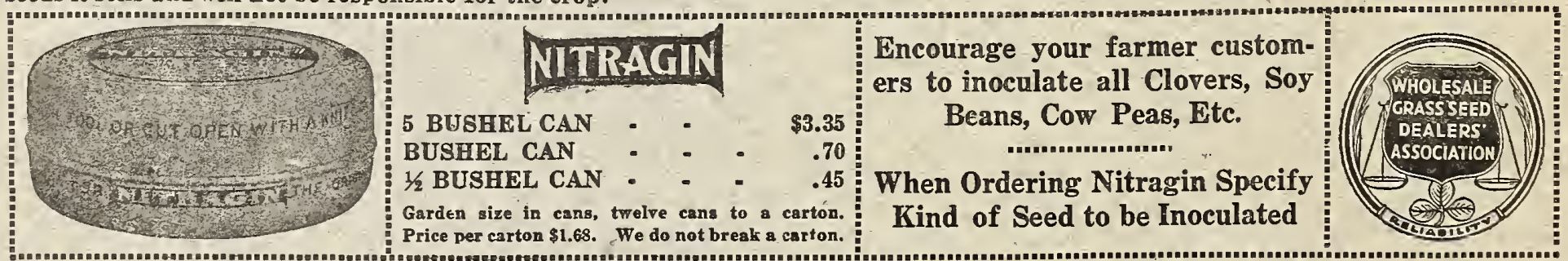





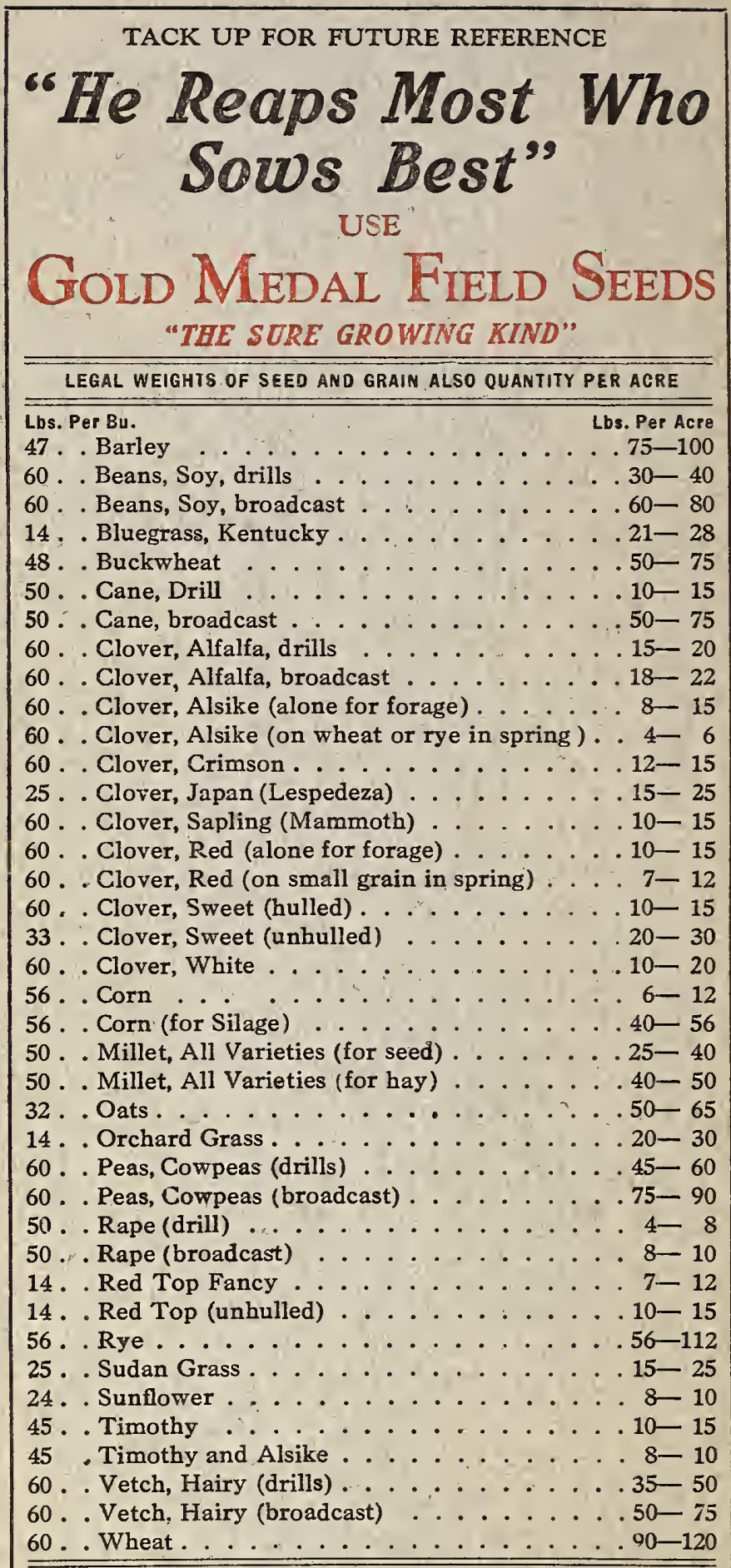

\section{Louisville Seed Company}

226 N. Fifteenth St.

LOUISVILLE, KENTUCKY 